International Journal of Pure and Applied Mathematics

Volume 105 No. 4 2015, 685-707

ISSN: 1311-8080 (printed version); ISSN: 1314-3395 (on-line version)

url: http://www.ijpam.eu

doi: http://dx.doi.org/10.12732/ijpam.v105i4.9

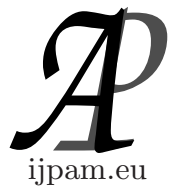

\title{
COMBINED EFFECT OF HEAT AND MASS TRANSFER ON MHD PERISTALTIC TRANSPORT OF A COUPLE STRESS FLUID IN A INCLINED ASYMMETRIC CHANNEL THROUGH A POROUS MEDIUM
}

\author{
A. Govindarajan ${ }^{1}$, E.P. Siva ${ }^{2}$, M. Vidhya ${ }^{3}$ \\ ${ }^{1,2}$ Department of Mathematics \\ SRM University \\ Kattankulathur, 603203, INDIA \\ ${ }^{3}$ Department of Mathematics \\ Sathyabama University \\ Sholinganallur, 600119, INDIA
}

\begin{abstract}
This paper describes the combined effect of heat and mass transfer on two dimensional peristaltic transport of a couple stress fluid in the presence of heat absorption in a inclined asymmetric channel. The fluid is electrically conducting through a porous medium in the presence of uniform magnetic field. Chemical reaction effect is included in the mathematical model. The systems of governing PDEs are solved analytically. The analytical solution is carried out under long wave length approximation. The solutions are obtained for the primary velocity of the fluid, pressure gradient, temperature and concentration of the fluid. The flow is found in the wave frame of reference moving with a velocity of the wave. The expression for average rise in pressure and frictional force and heat transfer coefficient are calculated by numerical integration and analyzed for various parameters by plotting the graphs and discussed in detail.
\end{abstract}

AMS Subject Classification: 76S05, 76T20, 74F10, 35K41

Key Words: couple stress fluid, peristaltic transport, heat transfer, MHD, mass transfer, inclined asymmetric channel

Received: August 23, 2015

(C) 2015 Academic Publications, Ltd. url: www.acadpubl.eu

$\S_{\text {Correspondence author }}$ 


\section{Introduction}

Peristalsis, a fundamental property of all parts of the tubular gut, is responsible for the propulsion of gastrointestinal contents along the gastrointestinal tract. Peristalsis is the movement of body fluids travelling through tubular organs continuously by contraction and relaxation due to change in pressure along the walls. This phenomenon is observed in the human body during the transport of lymph on lymphatic vessels, while swallowing food through the esophagus to the stomach, tube capillaries, vennules and arteries, the vasomotion of small blood vessels, the movement of feces in the large intestine, transport of chyme through the small intestine, the flow of semen in the male reproductive tract, the passage of urine from the kidneys through the urethra to the urinary bladder and the movement of ovum in the female fallopian tube. The examples cited above show the importance of peristalsis in the human body. Peristaltic phenomenon is used in biomedical instruments like heart-lung machines, dialysis machines, artificial heart and ortho machines and transport of toxic material waste inside the sanitary ducts. Peristaltic concept is used in nuclear industry to avoid contamination of the outside environment. Beginning with the first investigation of Latham [1], several theoretical and experimental attempts have been made to understand peristaltic action in different situations. Shapiro et al. [2] discussed the theoretical results for both plane and axisymmetric geometries. This has attracted several investigators to study the peristaltic transport under long wavelength and low Reynolds number approximation. Number of researchers [3-14,32] has discussed the peristaltic flow problems in different flow geometries. In particular, Hayat et al. [3] studied the peristaltic transport of a third-order fluid in a circular cylindrical tube. Wang et al [4] discussed the peristaltic motion of a Johnson-Segalman fluid in a planar channel. Rani and Sarojamma [5] have studied the peristaltic transport of a Casson fluid in an asymmetric channel. Peristaltic transport of a physiological fluid in an asymmetric porous channel in the presence of an external magnetic field was discussed by Misra et al [6]. Peristaltic motion of a power-law fluid in an asymmetric channel has described by Subba Reddy et al. [7]. ObaidUllahMehmood [8] has developed the mathematical model for dissipative heat transfer in Sisko fluid peristaltic flow through a cylindrical tube with nonlinear slip. Siva et al. [9] have analysed the effects of wall properties on MHD peristaltic flow of a dusty fluid through a porous medium. Govindarajan et al. [11] studied the 3D couette flow of dusty fluid with transpiration cooling. Govindarajan et al. [12] discussed the chemical reaction effects on unsteady MHD free convective flow in a rotating porous medium with mass transfer. Vidhya et al. [13] analyzed 
the chemical reaction effects on radiative MHD oscillatory flow in a porous channel with heat and mass transfer in an asymmetric channel. Siva et al [14] investigated the unsteady hydromagnetic flow of dusty viscous fluid in rotating channel with varying pressure gradient. Vidhya and Sundarammal [32] studied the effect of heat source parameter on laminar convection through porous medium between two vertical parallel plates.A very few investigators [10, 15-18] have analysed the peristaltic flow for different physical properties in inclined symmetric/asymmetric channel. Vajravelu et.al [10] analysed the peristaltic transport of a Herschel Bulkley fluid in an inclined tube. Haroun [15] have developed the mathematical model for non-linear peristaltic flow of a fourth grade fluid in an inclined asymmetric channel. Srinivas and Pushparaj [16] investigated the non-linear peristaltic transport in an inclined asymmetric channel. Kothandapani and Srinivas [17] extended their work to non-linear peristaltic transport of a Newtonian fluid in an inclined asymmetric channel through a porous medium. Nadeem and Akram [18] examined the influence of inclined magnetic field on peristaltic flow of a Williamson fluid model in an inclined symmetric or asymmetric channel. Mishra and Rao [19] contributed towards the study of peristaltic transport of a Newtonian fluid in an asymmetric channel. Hayat et al. [20] have developed the mathematical model for simultaneous effects of hall and convective conditions on peristaltic flow of couple-stress fluid in an inclined asymmetric channel. In particular, Siva and Govindarajan [21] discussed MHD peristaltic motion under the influence of heat/mass transfer with chemical reaction through a porous medium in an asymmetric channel.

The study of couple-stress fluids has plentiful applications in a number of processes that occur in industry such as solidification of liquid crystals, exotic lubricants, and colloidal solutions, cooling of metallic plate in a bath, the extrusion of polymer fluids, etc. Recently, peristaltic transport of non Newtonian fluid has been an important topic in the chemical field. A considerable amount the literature has been reported in [22-29]. Srivastava [22] developed the mathematical model for Peristaltic transport of a couple-stress fluid. Dulal et al. [23] studied a couple stress model of blood flow in the microcirculation. Mekheimer [24] investigated the effect of the induced magnetic field on peristaltic flow of a couple stress fluid. Vidhya et al. [25] studied the MHD peristaltic flow of a couple stress fluids permeated with suspended particles through a porous medium under long wavelength approximation. Few authors like Pandey [26], Mishra [27], Alsaedi [28], Sankad[29] (and all the reference therein) have studied the non Newtonian fluid. Few authors [30-31] discussed the heat and mass transfer for non Newtonian fluid. Hayat et al [30] studied the peristaltic flow of couplestress fluid with heat and mass transfer. Ramesh and Devakar [31] discussed 
the effects of heat and mass transfer on the peristaltic transport of MHD couple stress fluid through porous medium in a vertical asymmetric channel.

As far as our knowledge goes, the combined effect of heat and mass transfer on two dimensional peristaltic transport of a couple stress fluid in the presence of heat absorption in a inclined asymmetric channel has not been studied before. Therefore the main aim of this study is to construct a mathematical model to examine effect of heat and mass transfer on two dimensional peristaltic transport of a couple stress fluid in the presence of heat absorption in a inclined asymmetric channel. The fluid is electrically conducting through a porous medium in the presence of uniform magnetic field. A chemical reaction effect has been included in the mathematical model. The system of governing PDEs are solved analytically. The analytical solution is found under long wave length approximation. The solutions are obtained for the primary velocity of the fluid, pressure gradient, temperature and concentration of the fluid. The flow is found in the wave frame of reference moving with a velocity of the wave. The expression for average rise in pressure and frictional force has been calculated by numerical integration. Numerical calculations are carried for pressure rise, frictional force and heat transfer coefficient are analyzed by plotting the graphs and discussed in detail.

\section{Mathematical Analysis}

In the present paper, consider an incompressible viscous couple stress fluid in an inclined asymmetric channel through a porous medium. A fluid is conducting in the presence of an applied induced magnetic field $B_{0}$. The width of the channel is $d_{1}+d_{2}$. The medium is considered to be induced by a sinusoidal wave train with a wave speed $c$ along the length of the channel wall. The walls $y=H_{1}$ and $y=H_{2}$ maintained at the temperature $T_{0}$ and $T_{1}$ and with the concentration $C_{0}$ and $C_{1}$ respectively.

The channel walls are represented by

$$
\begin{gathered}
Y=H_{1}(X, t)=d_{1}+a_{1} \cos \frac{2 \pi}{\lambda}(X-c t) \\
Y=H_{2}(X, t)=-d_{2}-a_{2} \cos \left(\frac{2 \pi}{\lambda}(X-c t)+\phi\right)
\end{gathered}
$$

Where $a_{1}, a_{2}$ are the amplitudes of the waves, $\lambda$ is the wave length, the phase difference $\phi$ varies in the range $0 \leq \phi \leq \pi, \phi=0$ corresponds to the symmetric channel with waves out of phase and $\phi=\pi$ the waves are in the phase, and further $a_{1}, a_{2}, d_{1}, d_{2}$ and $\phi$ satisfies the condition 


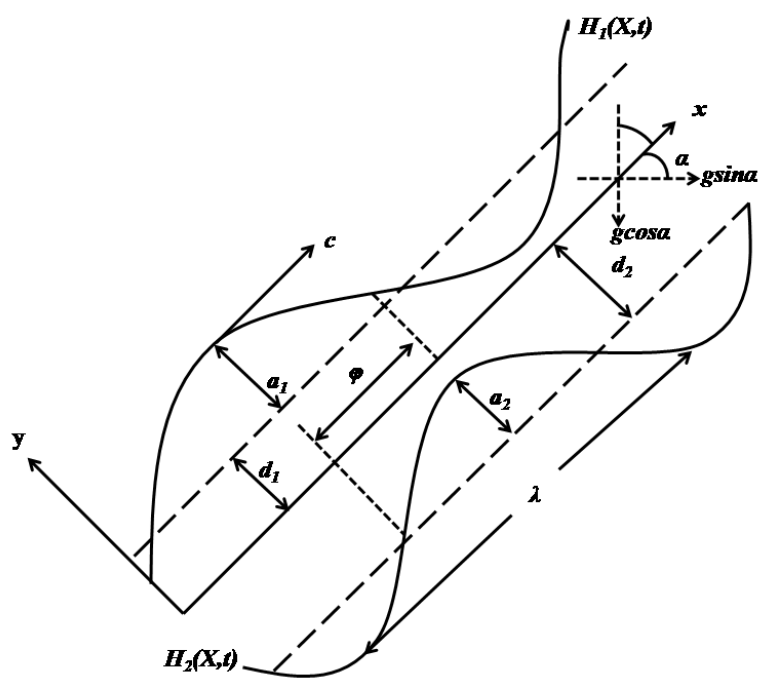

Fig. 1. Schematic diagram of 2- dimensional asymmetric channels

$$
a_{1}^{2}+b_{1}^{2}+2 a_{1} b_{1} \cos \phi \leq\left(d_{1}+d_{2}\right)^{2}
$$

For the incompressible viscous electrically conducting couple stress fluid through the porous medium, the governing equations of continuity, motion (including MHD, porous term), and energy are

$$
\begin{gathered}
\frac{\partial U}{\partial X}+\frac{\partial V}{\partial Y}=0 \\
\rho\left(\frac{\partial U}{\partial t}+U \frac{\partial U}{\partial X}+V \frac{\partial U}{\partial Y}\right)=-\frac{\partial P}{\partial X}+\mu \nabla^{2} U-\eta \nabla^{4} U-\frac{\mu}{k_{0}} U-\sigma^{*} B_{0}^{2} U \\
+\rho g \beta_{t}\left(T-T_{0}\right)+\rho g \beta_{c}\left(C-C_{0}\right)+\rho g \sin \alpha \\
\rho\left(\frac{\partial V}{\partial t}+U \frac{\partial V}{\partial X}+V \frac{\partial V}{\partial Y}\right)=-\frac{\partial P}{\partial Y}+\mu \nabla^{2} V-\eta \nabla^{4} V-\frac{\mu}{k_{0}} V-\rho g \cos \alpha \\
\rho C_{p}\left(\frac{\partial T}{\partial t}+U \frac{\partial T}{\partial X}+V \frac{\partial T}{\partial Y}\right)=K \nabla^{2} T+Q_{0} \\
\left(\frac{\partial C}{\partial t}+U \frac{\partial C}{\partial X}+V \frac{\partial C}{\partial Y}\right)=D_{m} \nabla^{2} C-K_{1} C
\end{gathered}
$$


where

$$
\nabla^{2}=\left(\frac{\partial^{2}}{\partial X^{2}}+\frac{\partial^{2}}{\partial Y^{2}}\right)
$$

In nature, the flow concept is unsteady in the fixed frame. To find out the steady analysis, defining a wave frame $(x, y)$ with velocity $c$ from the fixed frame $(X, Y)$, velocity components $u, v$ and the pressure $p$ by

$$
x=X-c t, y=Y, u=U-c, v=V \quad \text { and } \quad p(x)=P(X, t)
$$

Where $\rho$ is the density of the fluid, $\mu$ is the coefficient of viscosity, $U, V$ are the velocities in $X$ and $Y$ directions in the frame of reference, $P$ is the pressure in fixed frame, $k_{0}$ is the permeability of the porous medium, $g$ is the gravitational acceleration, $T$ is the temperature of the fluid, $\beta_{t}$ is the thermal expansion coefficient, $\beta_{c}$ is the concentration expansion coefficient, $\eta$ is the constant associated with couple stress, $\sigma^{*}$ is the electric conductivity coefficient, $B_{0}$ is the transverse magnetic field, $K$ is the thermal conductivity, $D_{m}$ is the mass diffusivity coefficient, $K_{1}$ is the first order chemical reaction rate, $C_{p}$ is the specific heat at the constant pressure, $Q_{0}$ is the constant heat addition/absorption.

Introducing the following non-dimensional variables:

$$
\begin{gathered}
\bar{x}=\frac{x}{\lambda}, \bar{y}=\frac{y}{d_{1}}, \bar{u}=\frac{u}{c}, \bar{v}=\frac{v}{c \delta}, \delta=\frac{d_{1}}{\lambda}, \bar{p}=\frac{d_{1}^{2} p}{\mu c \lambda}, \bar{t}=\frac{c t}{\lambda}, h_{1}=\frac{H_{1}}{d_{1}}, h_{2}=\frac{H_{2}}{d_{1}}, \\
d=\frac{d_{2}}{d_{1}}, a=\frac{a_{1}}{d_{1}}, b=\frac{a_{2}}{d_{1}}, R e=\frac{\rho c d_{1}}{\mu}, \theta=\frac{T-T_{0}}{T_{1}-T_{0}}, \sigma=\frac{C-C_{0}}{C_{1}-C_{0}}, \gamma=\frac{K_{1} d_{1}^{2}}{\mu}, \\
S^{2}=\frac{\mu_{1} d_{1}^{2}}{\eta}, F r=\frac{c^{2}}{g d_{1}^{2}}, G r=\frac{\rho g \beta_{t} d_{1}^{2}\left(T_{1}-T_{0}\right)}{c \mu}, G c=\frac{\rho g \beta_{c} d_{1}^{2}\left(C_{1}-C_{0}\right)}{c \mu}, \\
M^{2}=\frac{\sigma^{*} B_{0}^{2} d_{1}^{2}}{\mu}, \operatorname{Pr}=\frac{C_{p} \mu}{K}, S c=\frac{\mu}{\rho D_{m}}, k=\frac{k_{0}}{d_{1}^{2}}, \beta=\frac{Q_{0} d_{1}^{2}}{\left(T_{1}-T_{0}\right) \mu C_{p}} .
\end{gathered}
$$

Using these non dimensional variables in the basic equations one can obtain the non dimensional form, by dropping the bars, as

$$
\begin{gathered}
h_{1}=1+a \cos 2 \pi x, h_{2}=-d-b \cos (2 \pi x+\phi) \\
\frac{\partial u}{\partial x}+\frac{\partial v}{\partial y}=0
\end{gathered}
$$




$$
\begin{gathered}
\operatorname{Re}\left(u \frac{\partial u}{\partial x}+v \frac{\partial u}{\partial y}\right)=-\frac{\partial p}{\partial x}+\left(\delta^{2} \frac{\partial^{2} u}{\partial x^{2}}+\frac{\partial^{2} u}{\partial y^{2}}\right)-\frac{1}{S^{2}}\left(\delta^{4} \frac{\partial^{4} u}{\partial x^{4}}+\frac{\partial^{4} u}{\partial y^{4}}\right) \\
-\left(\frac{1}{k}+M^{2}\right)(u+1)+G r \theta+G c \sigma+\frac{R e}{F r} \sin \alpha \\
\operatorname{Re} \delta^{3}\left(u \frac{\partial v}{\partial x}+v \frac{\partial v}{\partial y}\right)=-\frac{\partial p}{\partial y}+\delta^{2}\left(\delta^{2} \frac{\partial^{2} v}{\partial x^{2}}+\frac{\partial^{2} v}{\partial y^{2}}\right) \\
-\frac{\delta^{2}}{S^{2}}\left(\delta^{4} \frac{\partial^{4} v}{\partial x^{4}}+\frac{\partial^{4} v}{\partial y^{4}}\right)-\frac{\delta^{2} v}{k}-\frac{\operatorname{Re} \delta}{F r} \cos \alpha \\
\operatorname{RePr}\left(u \frac{\partial \theta}{\partial x}+v \frac{\partial \theta}{\partial y}\right)=\left(\delta^{2} \frac{\partial^{2} \theta}{\partial x^{2}}+\frac{\partial^{2} \theta}{\partial y^{2}}\right)+\beta \\
\operatorname{Re} \delta\left(u \frac{\partial \sigma}{\partial x}+v \frac{\partial \sigma}{\partial y}\right)=\frac{1}{S c}\left(\delta^{2} \frac{\partial^{2} \sigma}{\partial x^{2}}+\frac{\partial^{2} \sigma}{\partial y^{2}}\right)-\gamma \sigma-c_{1}^{*}
\end{gathered}
$$

Where

$$
c_{1}^{*}=\frac{K_{1} d_{1}^{2} C_{0}}{\mu\left(C_{1}-C_{0}\right)}
$$

Where $R e$ is the Reynolds number, $\delta$ is the wave number, $u$ and $v$ are velocity components in $x$ and $y$ directions respectively, $p$ is the the dimensionless pressure, $M$ is the Hartmann number, $k$ is the porosity parameter, $S$ is the couple stress parameter, $G r$ is the local temperature Grashof number, $G c$ is the local mass Grashof number, $F r$ is the Fraude number, $\alpha$ is the inclination of the channel, $\operatorname{Pr}$ is the Prandtl number, $\theta$ is the dimensionless temperature of the fluid, $\sigma$ is the dimensionless concentration of the fluid, $S c$ is the Schmidt number, $\gamma$ is the the chemical reaction parameter and $\beta$ is the Non Dimensional heat source/sink parameter.

Using approximation of the long wavelength $(\delta<<1)$ and neglecting the wave number, one we can find from (10)to (13) that

$$
\begin{gathered}
-\frac{\partial p}{\partial x}+\left(\frac{\partial^{2} u}{\partial y^{2}}\right)-\frac{1}{S^{2}}\left(\frac{\partial^{4} u}{\partial y^{4}}\right)-\left(\frac{1}{k}+M^{2}\right)(u+1)+G r \theta+G c \sigma+\frac{R e}{F r} \sin \alpha=0 \\
\frac{\partial p}{\partial y}=0 \\
\left(\frac{\partial^{2} \theta}{\partial y^{2}}\right)+\beta=0
\end{gathered}
$$




$$
\frac{1}{S c}\left(\frac{\partial^{2} \sigma}{\partial y^{2}}\right)-\gamma \sigma-c_{1}^{*}=0
$$

The corresponding boundary conditions are

$$
\begin{aligned}
& u=-1 \quad \text { at } \quad y=h_{1} \quad \text { and } \quad y=h_{2} \\
& \theta=0 \quad \text { at } \quad y=h_{1} \text { and } \quad \theta=1 \quad \text { at } \quad y=h_{2} \\
& \sigma=0 \quad \text { at } \quad y=h_{1} \text { and } \quad \sigma=1 \quad \text { at } \quad y=h_{2}
\end{aligned}
$$

In laboratory frame, the dimensional volume flow rate is

$$
\bar{Q}=\int_{H_{2}(X, t)}^{H_{1}(X, t)} U(X, Y, t) d Y
$$

In which $H_{1}$ and $H_{2}$ are functions of $X$ and t. The above expression in the wave frame becomes

$$
q=\int_{h_{2}}^{h_{1}} u(x, y) d y
$$

Where $h_{1}$ and $h_{2}$ are the functions of $\mathrm{x}$ alone.

\section{Solution}

Solving Equation (14)-(17) using boundary conditions (18) -(20), the solution is obtained as

$$
\begin{gathered}
\theta=-\frac{\beta}{2} y^{2}+c_{1} y+c_{2} \\
\sigma=c_{3} \cosh z y+c_{4} \sinh z y-\frac{c_{1}^{*}}{\gamma} \\
u=c_{5} \cosh A y+c_{6} \sinh A y+c_{7} \cosh B y+c_{8} \sinh B y+L_{3} y^{2}+L_{4} y \\
+L_{5} \cosh z y+L_{6} \sinh z y+L_{1}
\end{gathered}
$$

The dimensionless time mean flow rate in the wave frame is $Q=q+1+d$.

The pressure rise/wave length is

$$
\Delta P=\int_{0}^{1}\left(\frac{d p}{d x}\right) d x
$$


Where the pressure gradient is

$$
\frac{d p}{d x}=\frac{q-L_{33}}{L_{34}}
$$

The friction forces at $y=h_{1}$ and $y=h_{2}$ are

$$
F_{\lambda 1}=\int_{0}^{1}-h_{1}^{2}\left(\frac{d p}{d x}\right) d x, F_{\lambda 2}=\int_{0}^{1}-h_{2}^{2}\left(\frac{d p}{d x}\right) d x
$$

The heat transfer coefficient at the right hand wall is

$$
Z_{h 1}=\left(\frac{\partial \theta}{\partial y} \cdot \frac{\partial h_{1}}{\partial x}\right)_{y=h_{1}}=2 a \pi\left(\beta y-c_{1}\right) \sin 2 \pi x
$$

Where $A=\sqrt{\frac{S}{2}\left(S+\sqrt{S^{2}-4 H^{2}}\right)}, B=\sqrt{\frac{S}{2}\left(S-\sqrt{S^{2}-4 H^{2}}\right)}, z=\sqrt{\gamma \cdot S c}$, $H=\sqrt{M^{2}+\frac{1}{k}}$

\section{Graphical Illustration}

To study the graphical and physical significance of various flow parameters of couple stress fluid on MHD peristaltic flow in the inclined asymmetric channel, Fig.2 to Fig. 7 have been plotted.

\subsection{Flow Characteristics}

To see the effects of Hartmann number $(M)$, porous permeability parameter $(k)$, phase shift $(\phi)$, local temperature Grashof number $(G r)$, inclination of the channel $(\alpha)$, Froude number $(F r)$, chemical reaction parameter $(\gamma)$, Schmidt number $(S c)$, Couple stress parameter $(S)$ on the velocity profile $(u)$, is graphed Fig.2 for fixed values of $a=0, b=0.4, x=0.3, d=1.12, \phi=0, R e=1, d p / d x$ $=1, G c=5, c_{1}^{*}=1, \alpha=0, F r=0.2, G r=5, \gamma=0.2, S c=0.2, S=0.2, k=$ $0.2, M=2$. Fig.2.a is plotted to see the variation of axial velocity for different values of $M$. It is observed that the velocity profiles are parabolic in nature. It is considered from this figure that the axial velocity profile decreases when $M$ increases. This seems realistic, because the magnetic field acts in the transverse direction to the flow and magnetic force resists the flow. The same behavior can be seen with increasing $S c$ and opposite behavior has seen for $S$ ( see Fig.2.b and Fig.2.c). Fig.2.d shows that the axial velocity profile on different values of 
$\alpha$. Moreover, the velocity increases with increasing $\alpha$ in the middle part of the channel. Fig.2.e displays the axial velocity for different value of Fr. It is viewed that the axial velocity increases with increase in Fr. Fig.2.f is drawn to study the effect of $G r$ for the axial velocity distribution. It is noticed that the axial velocity increases with increasing Gr. Figs.2.g and Fig.2.h shows that the axial velocity profile increases with increasing $k$ and opposite behavior has seen for $\gamma$.

\subsection{Pumping Characteristics}

Fig.3 illustrates the variation of pressure gradient over one wave length $x \in[0,1]$ for the fixed values of $a=0.5, b=0.5, d=1, \phi=\pi / 4, R e=2, G c=0.5$, $c_{1}^{*}=1, \alpha=0, F r=0.3, G r=0.5, \gamma=0.2, S c=0.2, S=1.5, k=0.2, M$ $=2, q=-3$. The effects of $M, \alpha, \phi, \beta, G r, k, S$ and $q$ on pressure gradient are displayed in Fig.3.a to Fig.3.h. It has been observed from Fig.3.a that increasing in $M$ increases the pressure gradient. This result suggests that the fluid pressure can be controlled by the application of suitable magnetic field strength. More over this phenomenon is useful during surgery and critical operation to control excessive bleeding. It is also noted that increasing of $\alpha$, $\beta$ and $G r$ increases the pressure gradient (see Fig.3.b, Fig.3.d, Fig.3.e). It is observed From Fig.3.f to Fig.3.h, that with the increasing of $k, S$ and $q$, the pressure gradient decreases. It is observed that, in the wider part of the channels $x \in[0,0.25]$ and $x \in[0.75,1]$, the pressure gradient is small, so the flow can be easily passed without the imposition of large pressure gradient. However, in the narrow part of the channel $x \in[0.25,0.75]$ the pressure gradient is large,(i.e), much larger pressure gradient is needed to maintain the same given volume flow rate.

The effects of $\beta, G r, \alpha, M, S, k$ on the dimensionless pressure drop $\Delta P$ against $Q$ (time averaged flux) are shown in Fig.4 for the fixed values of $a=$ $0.5, b=0.5, d=1, \phi=\pi / 4, R e=2, G c=0.5, c_{1}^{*}=1, \alpha=0, F r=0.2, G r$ $=0.5, \gamma=0.2, S c=0.2, S=1.5, k=0.2, M=2, q=-3$. The whole region split into four segments. The upper right hand quadrant (I quadrant) denotes the peristaltic pumping region $(Q<0$ and $\Delta P>0)$. The second quadrant is such that $\Delta P<0$ and $Q>0$ is called as augmented flow. Free pumping region corresponds to $\Delta P=0$. In this region, the positive value of $Q$ is entirely due to the peristalsis after overcoming the pressure difference. The region where $\Delta P>0$ and $Q<0$ is known retrograde or backward pumping. It shows that there is a linear relation between $\Delta P$ and $Q$. Moreover, it is noted that region of peristaltic pumping increases with an increase of $\beta$ (Fig.4.a), Gr (Fig.4.b), 


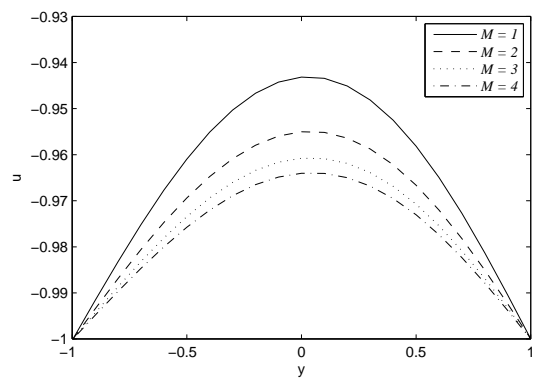

(a) Fig.2.a Effect of $M$ on $u$

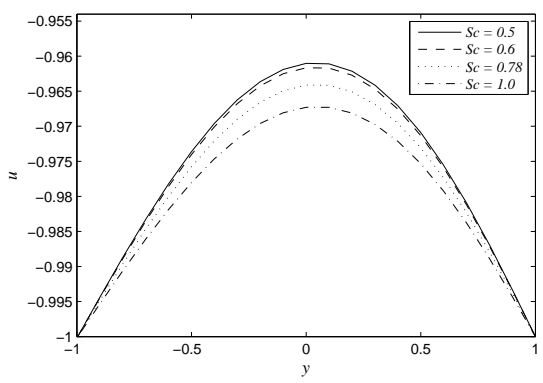

(b) Fig.2.b Effect of $S c$ on $u$

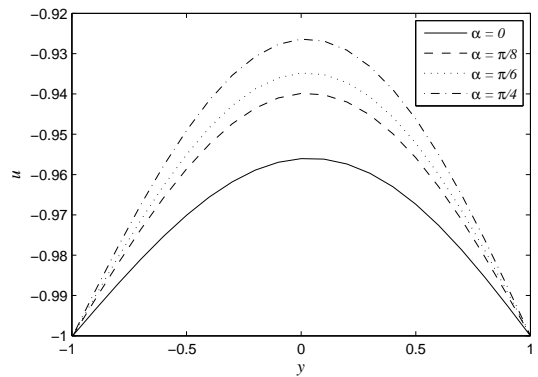

(d) Fig.2.d Effect of $\alpha$ on $u$

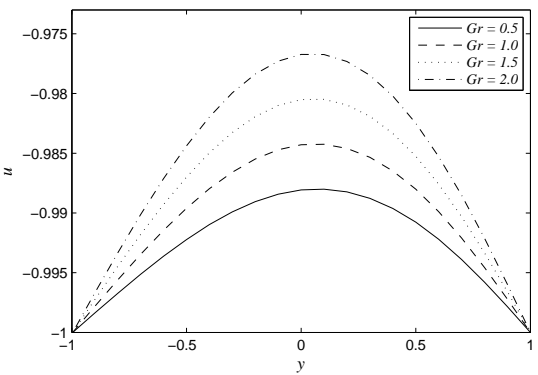

(f) Fig.2.f Effect of $G r$ on $u$

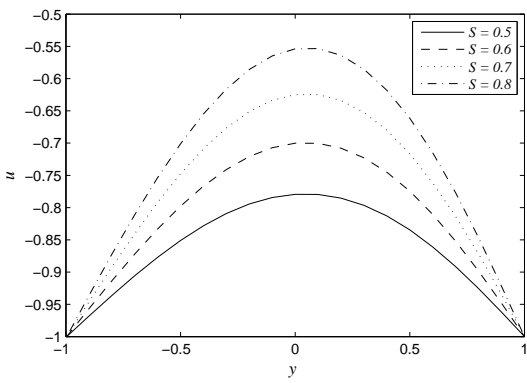

(c) Fig.2.c Effect of $S$ on $u$

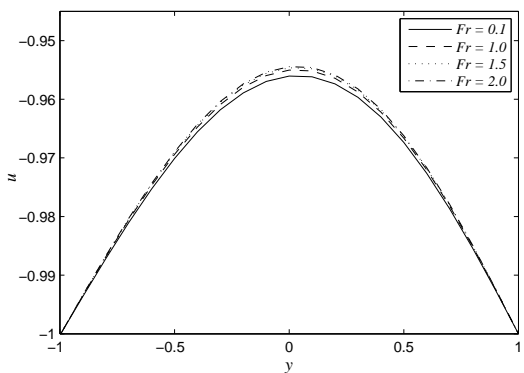

(e) Fig.2.e Effect of $F r$ on $u$

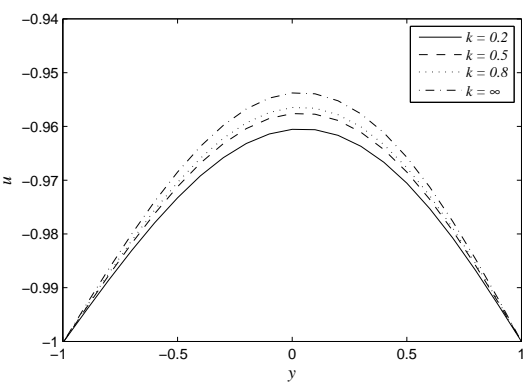

(g) Fig.2.g Effect of $k$ on $u$ 


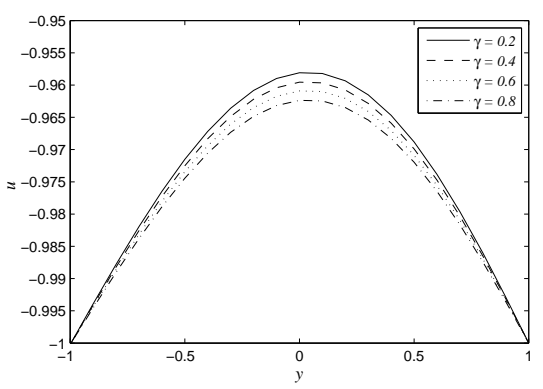

(a) Fig.2.h Effect of $\gamma$ on $u$

$\alpha$ (Fig.4.c). From Fig.4.d, it is clear that with the increasing value of $M$ in the augmented pumping and free pumping regions, the pumping decreases in the peristaltic pumping region. The pumping increases up to a critical value of $Q$ and decreases after the critical value and in the retrograde pumping region the pumping increases. It is observed from Fig.4.f that, with the increasing of $k$, the behavior is quite opposite with $M$. The same behavior observed from Fig.4.e

The effects of $M, k$ and $F r$ on $F_{\lambda 1}$ and $F_{\lambda 2}$ are plotted in Fig.5 and Fig.6 respectively. The figures in LHS (Fig.5.a, Fig.5.b, Fig.5.c), represents the variation of the frictional force at left wall of the channel $F_{\lambda 1}$ and the figures in RHS (Fig.6.a, Fig.6.b, Fig.6.c) depicts the variation of the frictional at the right wall of the channel $F_{\lambda 2}$. This phenomenon is quite opposite to the pumping characteristic. The frictional force $F_{\lambda 1}, F_{\lambda 2}$ are increases by increasing Hartmann number $M$ when $Q>0$. It is observed that the opposite behavior has seen for the permeability parameter $k . F_{\lambda 1}$ and $F_{\lambda 2}$ increases by increasing $F r$ after a certain value of $Q$.

\subsection{Heat and Mass Transfer Characteristics}

Fig.7.a and Fig.7.b indicates the effect of heat transfer and Heat transfer coefficient on the peristaltic transport for various values of $\beta$. Fig.7.a depicts that the temperature increases with $\beta$. It is observed from Fig.7.b that, due to peristalsis, the heat transfer coefficient is an oscillatory behavior has seen for the Heat source parameter $\beta$ and also noticed that absolute values of heat transfer coefficient increases with increasing $\beta$. Fig.7.c and Fig.7.d shows the behavior of concentration profile for the various values of $S c$ and $\gamma$. It has been observed that the concentration profile decreases by increasing both $S c$ and $\gamma$. 


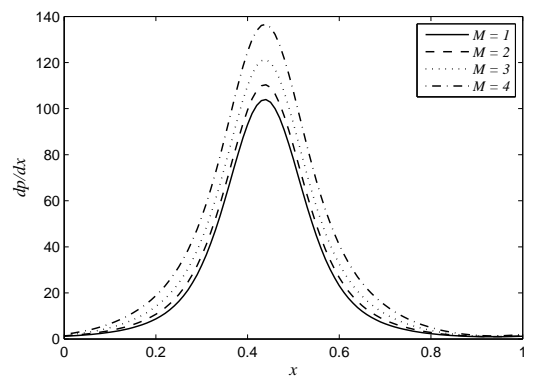

(a) Fig.3.a Effect of $M$ on $d p / d x$

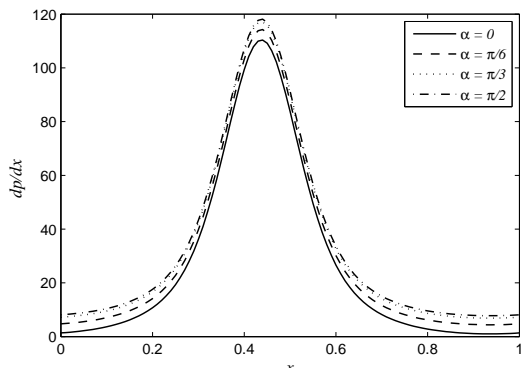

(b) Fig.3.b Effect of $\alpha$ on $d p / d x$

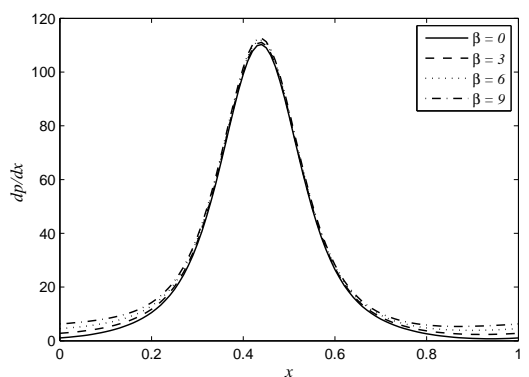

(d) Fig.3.d Effect of $\beta$ on $d p / d x$

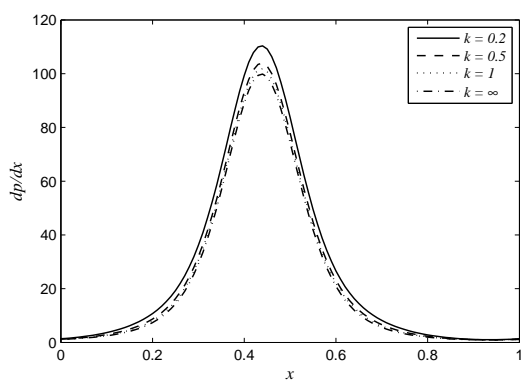

(f) Fig.3.f Effect of $k$ on $d p / d x$

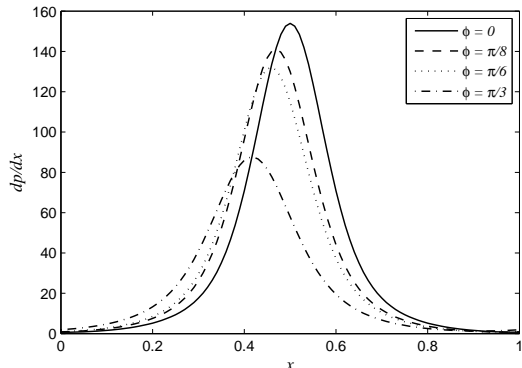

(c) Fig.3.c Effect of $\phi$ on $d p / d x$

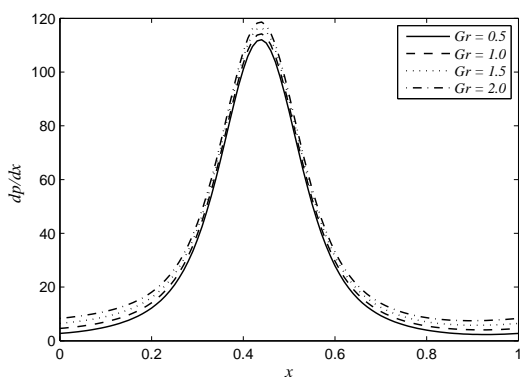

(e) Fig.3.e Effect of $G r$ on $d p / d x$

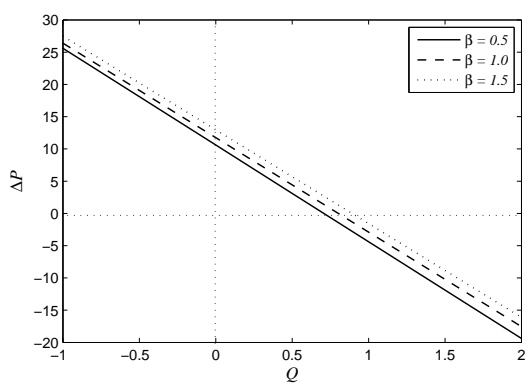

(g) Fig.4.a Effect of $\beta$ on $\Delta P$ 


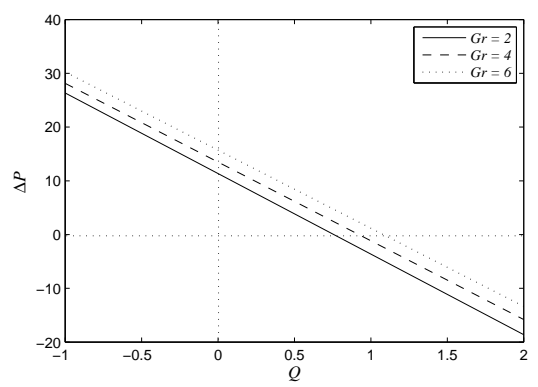

(a) Fig.4.b Effect of $G r$ on $\Delta P$

\section{Concluding Remarks}

The primary goal of this study is to analyze the combined effect of heat and mass transfer on two dimensional peristaltic transport of a couple stress fluid in the presence of heat absorption in a inclined asymmetric channel. The fluid is electrically conducting through a porous medium in the presence of uniform magnetic field. Chemical reaction effect has been included in the mathematical model. The systems of governing PDEs are solved analytically. The analytical solution is carried out under long wave length approximation. Analytical solution have been developed for axial velocity, pressure gradient, pressure drop, frictional force, temperature, concentration and heat transfer coefficient. The features of the flow characteristics are analyzed by plotting graphs and discussed in detail. The following points are made from the present study:

1. The axial velocity profile decreases with an increase in $M, S c$ and $\gamma$ whereas, it increases with the increase in $S, \alpha, F r, G r$ and $k$.

2. Axial pressure gradient increases with an increase in $M, \alpha, \beta$ and $G r$. It is also observed that pressure gradient decreases with an increase in permeability parameter $k$.

3. It is observed that the Magneto hydrodynamic effects in free and augmented pumping regions are different than the peristaltic pumping region. The same effect has observed for $S$.

4. The pressure rise increases in all the regions with an increase in $\alpha, G r$ and $\beta$.

5. Frictional force has the opposite behavior compared with pressure rise. 


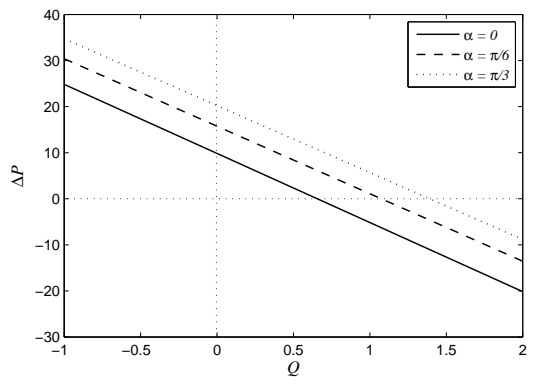

(a) Fig.4.c Effect of $\alpha$ on $\Delta P$

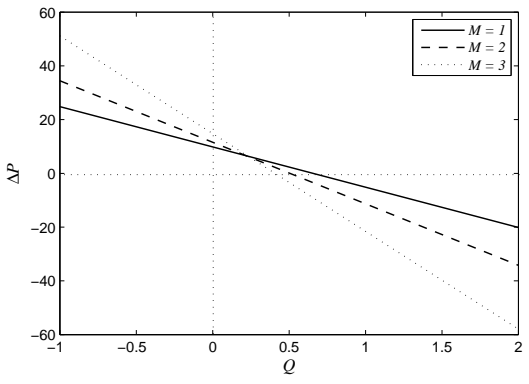

(b) Fig.4.d Effect of $M$ on $\Delta P$

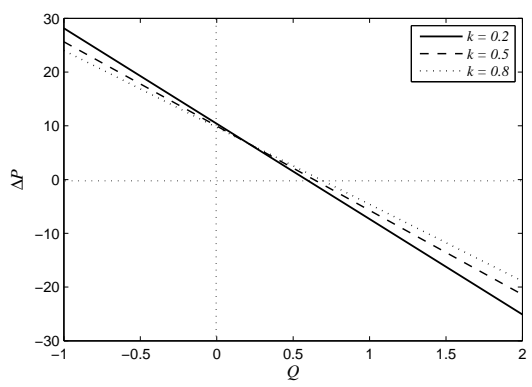

(d) Fig.4.f Effect of $k$ on $\Delta P$

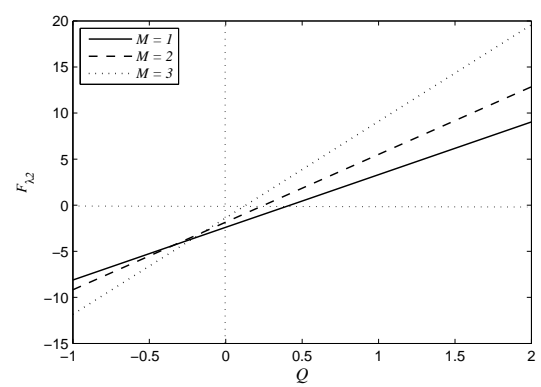

(f) Fig.6.a Effect of $M$ on $F_{\lambda 2}$

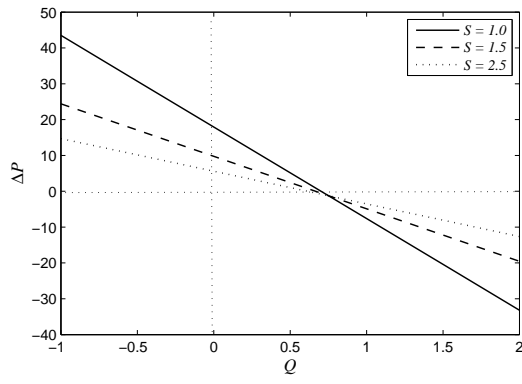

(c) Fig.4.e Effect of $S$ on $\Delta P$

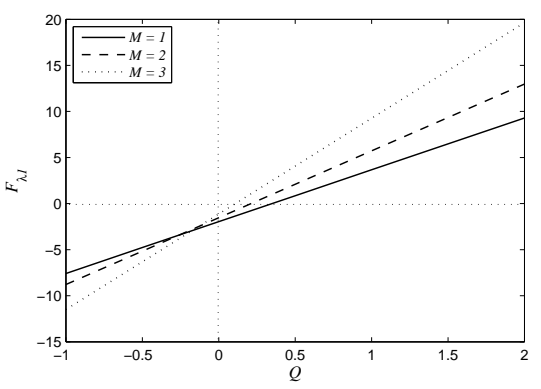

(e) Fig.5.a Effect of $M$ on $F_{\lambda 1}$

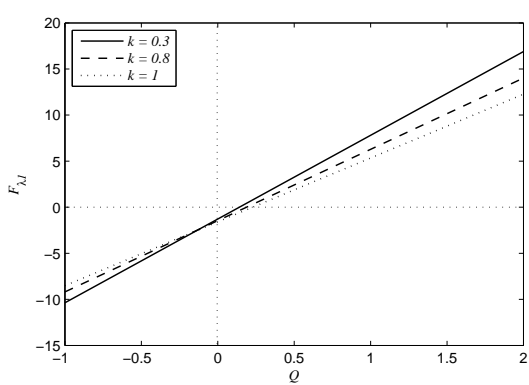

(g) Fig.5.b Effect of $k$ on $F_{\lambda 1}$ 


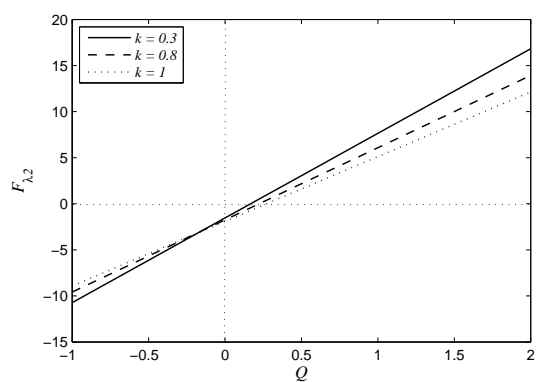

(a) Fig.6.b Effect of $k$ on $F_{\lambda 2}$

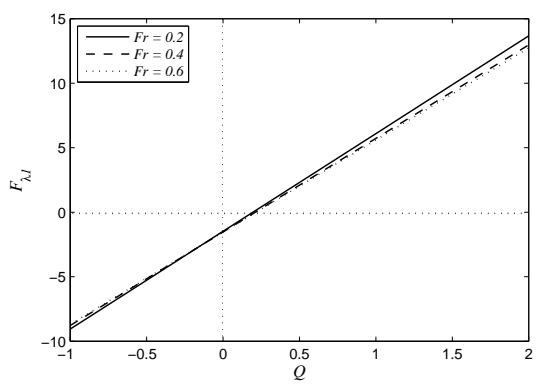

(a) Fig.5.c Effect of $F r$ on $F_{\lambda 1}$

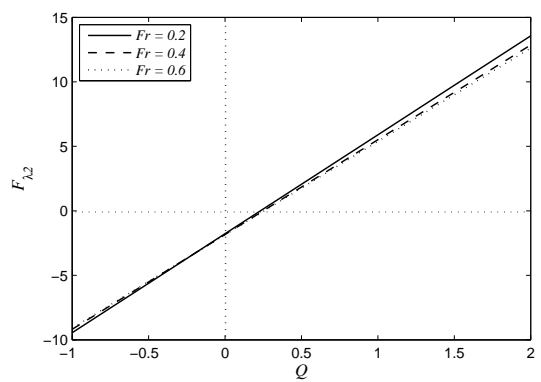

(b) Fig.6.c Effect of $F r$ on $F_{\lambda 2}$ 


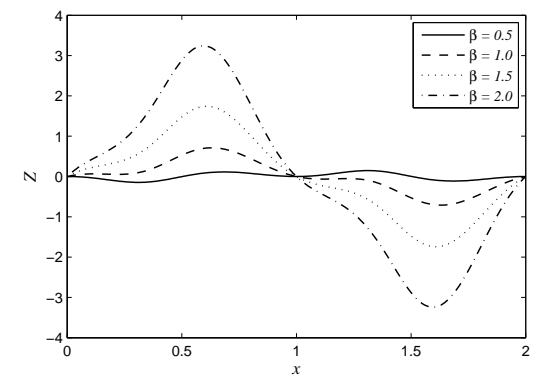

(a) Fig.7.a Effect of $\beta$ on Heat transfer coefficient for $a=0.5, b=0.5, d=1, \phi=$ $\pi / 4$
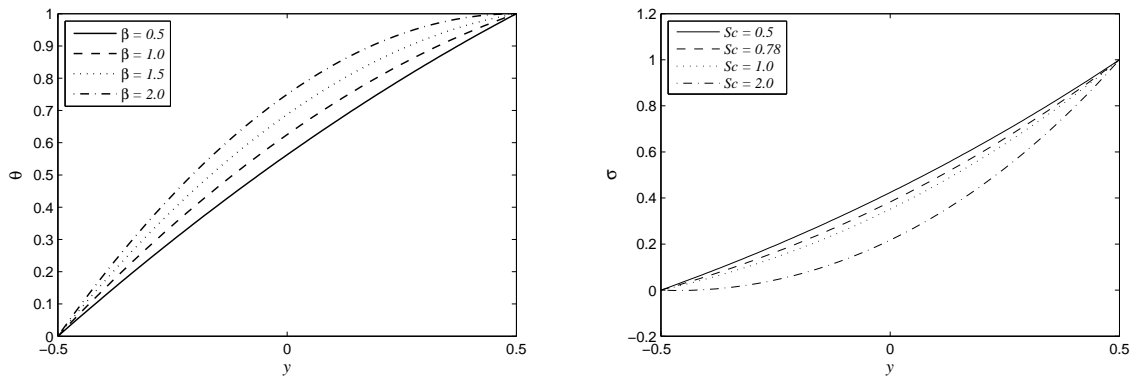

(b) Fig.7.b Effect of $\beta$ on $\theta$ for $a=0.5, b$ (c) Fig.7.c Effect of $S c$ on $\sigma$ for $a=0.5, b$ $=0.5, d=1, \phi=\pi / 4$ $=0.5, d=1, \phi=0, \gamma=0.5, c_{1}^{*}=1$

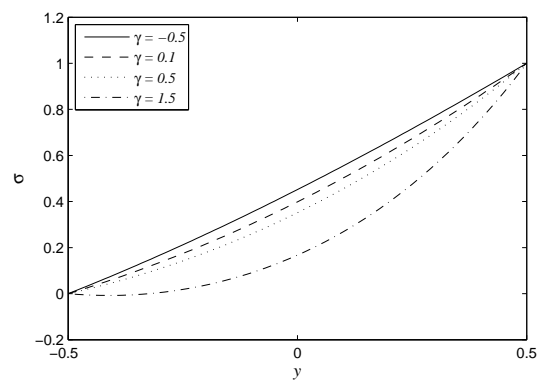

(d) Fig.7.c Effect of $\gamma$ on $\sigma$ for $a=0.5, b$

$=0.5, d=1, \phi=0, S c=0.2, c_{1}^{*}=1$ 
6. The heat transfer coefficient shows an oscillatory behavior for $\beta$. It is also noticed that absolute values of heat transfer coefficient increases with increasing $\beta$.

7. Concentration profile decreases by increasing both $S c$ and $\gamma$.

\section{Appendix}

$$
\begin{aligned}
& c_{1}=\frac{1+\frac{\beta}{2}\left(h_{1}^{2}-h_{2}^{2}\right)}{h_{1}-h_{2}}, \\
& c_{2}=1+\frac{\beta}{2} h_{1}^{2}-c_{1} h_{1}, \\
& c_{3}=\frac{\frac{c_{1}^{*}}{\gamma}-c_{4} \sinh z h_{2}}{\cosh z h_{2}}, \\
& c_{4}=\frac{\left(1+\frac{c_{1}^{*}}{\gamma}\right) \cosh z h_{2}-\frac{c_{1}^{*}}{\gamma} \cosh z h_{1}}{\sinh z\left(h_{1}-h_{2}\right)}, \\
& L_{2}=z^{4}-S^{2} z^{2}+S^{2} H^{2} \text {, } \\
& L_{3}=-\frac{G r \beta}{2 H^{2}} \text {, } \\
& L_{1}=\frac{G r}{H^{2}}\left(c_{2}+\frac{\beta}{H^{2}}\right)+\frac{\frac{R e}{F r} \sin \alpha-\frac{d p}{d x}-H^{2}-\frac{c_{1}^{*} G c}{\gamma}}{H^{2}}, \\
& L_{4}=-\frac{c_{1} G r}{H^{2}} \text {, } \\
& L_{5}=-\frac{c_{3} S^{2} G c}{L_{2}}, \\
& L_{6}=-\frac{c_{4} S^{2} G c}{L_{2}}, \\
& m_{1}=\cosh A h_{1} \text {, } \\
& m_{2}=\sinh A h_{1} \text {, } \\
& m_{3}=\cosh B h_{1} \text {, } \\
& m_{4}=\sinh B h_{1} \text {, } \\
& m_{5}=\cosh z h_{1} \text {, } \\
& m_{6}=\sinh z h_{1} \text {, }
\end{aligned}
$$




$$
\begin{aligned}
& n_{1}=\cosh A h_{2}, \\
& n_{2}=\sinh A h_{2} \text {, } \\
& n_{3}=\cosh B h_{2} \text {, } \\
& n_{4}=\sinh B h_{2} \text {, } \\
& n_{5}=\cosh z h_{2} \text {, } \\
& n_{6}=\sinh z h_{2} \text {, } \\
& G=-\left(2 L_{3}+L_{5} z^{2} m_{5}+L_{6} z^{2} m_{6}\right), \\
& E=-\left(1+L_{3} h_{1}^{2}+L_{4} h_{1}+L_{5} m_{5}+L_{6} m_{6}+L_{1}\right) \text {, } \\
& I=-\left(2 L_{3}+L_{5} z^{2} n_{5}+L_{6} z^{2} n_{6}\right), \\
& F=-\left(1+L_{3} h_{2}^{2}+L_{4} h_{2}+L_{5} n_{5}+L_{6} n_{6}+L_{1}\right) \text {, } \\
& c_{8}=\frac{n_{3}\left(A^{2} E-G\right)-m_{3}\left(A^{2} F-I\right)}{\left(A^{2}-B^{2}\right)\left(m_{4} n_{3}-n_{4} m_{3}\right)} \text {, } \\
& c_{7}=\frac{A^{2} F-I}{\left(A^{2}-B^{2}\right) n_{3}}-\frac{n_{4} c_{8}}{n_{3}} \text {, } \\
& c_{5}=\frac{I-A^{2} n_{2} c_{6}-B^{2} n_{3} c_{7}-B^{2} n_{4} c_{8}}{A^{2} n_{1}}, \\
& c_{6}=\frac{n_{1} G-m_{1} I-B^{2}\left(m_{3} n_{1}-n_{3} m_{1}\right) c_{7}-B^{2}\left(m_{4} n_{1}-n_{4} m_{1}\right) c_{8}}{A^{2}\left(m_{2} n_{1}-n_{2} m_{1}\right)}, \\
& L_{7}=\frac{G r}{H^{2}}\left(c_{2}+\frac{\beta}{H^{2}}\right)+\frac{\frac{R e}{F r} \sin \alpha-H^{2}-\frac{c_{1}^{*} G c}{\gamma}}{H^{2}}, \\
& L_{10}=\frac{n_{3} A^{2}}{H^{2}} \text {, } \\
& L_{11}=-\frac{m_{3} A^{2}}{H^{2}} \\
& L_{8}=-A^{2}\left(1+L_{3} h_{1}^{2}+L_{4} h_{1}+L_{5} m_{5}+L_{6} m_{6}+L_{7}\right)-G, \\
& L_{9}=-A^{2}\left(1+L_{3} h_{2}^{2}+L_{4} h_{2}+L_{5} n_{5}+L_{6} n_{6}+L_{7}\right)-F \text {, } \\
& L_{12}=\left(A^{2}-B^{2}\right)\left(m_{4} n_{3}-n_{4} m_{3}\right) \text {, } \\
& L_{13}=\left(n_{4} L_{8}-m_{3} L_{9}\right) \text {, } \\
& L_{14}=L_{10}+L_{11} \text {, } \\
& L_{15}=\frac{L_{9}}{n_{3}\left(A^{2}-B^{2}\right)}-\frac{n_{4} L_{13}}{n_{3} L_{12}}, \\
& L_{16}=\frac{A^{2} L_{9}}{H^{2} n_{3}\left(A^{2}-B^{2}\right)}-\frac{n_{4} L_{14}}{n_{3} L_{12}} \text {, }
\end{aligned}
$$




$$
\begin{aligned}
& L_{17}=\frac{L_{13}}{L_{12}}, \\
& L_{18}=\frac{14}{L_{12}}, \\
& L_{19}=B^{2}\left(m_{3} n_{1}-n_{3} m_{1}\right), \\
& L_{20}=B^{2}\left(m_{4} n_{1}-n_{4} m_{1}\right), \\
& L_{21}=n_{1} G-m_{1} I \text {, } \\
& L_{22}=A^{2}\left(m_{2} n_{1}-n_{2} m_{1}\right) \text {, } \\
& L_{23}=\frac{L_{21}-L_{19} L_{15}-L_{20} L_{17}}{L_{22}} \text {, } \\
& L_{24}=\frac{L_{19} L_{16}+L_{20} L_{18}}{L_{22}}, \\
& L_{25}=\frac{I-A^{2} n_{2} L_{23}-B^{2} n_{3} L_{15}-B^{2} n_{4} L_{17}}{A^{2} n_{1}}, \\
& L_{26}=\frac{A^{2} n_{2} L_{24}+B^{2} n_{3} L_{16}+B^{2} n_{4} L_{18}}{A^{2} n_{1}}, \\
& L_{27}=\frac{m_{2}-n_{2}}{A}, \\
& L_{28}=\frac{m_{1}-n_{1}}{A}, \\
& L_{29}=\frac{m_{4}-n_{4}}{B}, \\
& L_{30}=\frac{m_{3}-n_{3}}{B}, \\
& L_{31}=\frac{L_{3}\left(h_{1}^{3}-h_{2}^{3}\right)}{3}, \\
& L_{33}=L_{25} L_{27}+L_{23} L_{28}+L_{15} L_{29}+L_{17} L_{30}+L_{31} \text {, } \\
& L_{32}=-\frac{\left(h_{1}-h_{2}\right)}{H^{2}} \text {, } \\
& L_{34}=L_{26} L_{27}+L_{24} L_{28}+L_{16} L_{29}+L_{18} L_{30}+L_{32} \text {. }
\end{aligned}
$$

\section{References}

[1] T.W.Latham, Fluid motion in Peristaltic pump, MS thesis, Mit Cambridge, (1966).

[2] A.H.Shapiro, M.Y.Jaffrin and S.L.Weinberg, Peristaltic pumping with long 
wavelength at low Reynolds number, Journal of fluid mechanics, 37, No.4(1969), 799-825.

[3] T.Hayat, Y.Wang, A.M.Siddiqui, K.Hutter and S.Asghar, Peristaltic transport of a third-order fluid in a circular cylindrical tube Models, Mathematical models and Methods in Applied Science, 12, No.12(2002), 1691-1706.

[4] T.Hayat, Y.Wang, A.M.Siddiqui and K.Hutter, Peristaltic motion of a Johnson-Segalman fluid in a planar channel, Mathematical Problems in Engineering, 2003, No.1(2003), 1-23.

[5] P.N.Rani, G.Sarojamma, Peristaltic transport of a Casson fluid in an asymmetric channel, Australas Physics Engineering Science Med., 27, No.2(2004), 49-59.

[6] J.C.Misra, S.Maiti and G.C.Shit, Peristaltic transport of a physiological fluid in an asymmetric porous channel in the presence of an external magnetic field, Journal of Mechanics in Medicine and Biology, 08, No.4(2008), 507-525.

[7] M.V.Subba Reddy, A.Ramachandra Rao and S.Sreenadh, Peristaltic motion of a power-law fluid in an asymmetric channel, International Journal of Non-Linear Mechanics, 42, No.10(2007), 1153-1161.

[8] Md.Obaidullah, N.Mustapha, S.Shafie and M.Qasim, Dissipative heat transfer in Sisko fluid peristaltic flow through a cylindrical tube with nonlinear slip, Heat Transfer Research, 46, No.7(2015), 643-656.

[9] E.P.Siva, A.Govindarajan and M.Vidhya, Analysis on the effects of wall properties on MHD peristaltic flow of a dusty fluid through a porous medium, International Journal of Pure and Applied Mathematics, 102, No.2(2015), 247-263.

[10] K.Vajravelu, S.Sreenadh and V.Ramesh Babu, Peristaltic transport of a Herschel Bulkley fluid in an inclined tube, International Journal of NonLinear Mechanics , 40, No.1(2005), 83-90.

[11] A.Govindarajan, V.Ramamurthy and K.Sundarammal, 3D couette flow of dusty fluid with transpiration cooling, Journal of Zhejiang University SCIENCE A, 8, No.2(2007), 313-322.

[12] A.Govindarjan, A.J.chamkha, K.Sundarammal and M.vidhya, Chemical reaction effects on unsteady MHD free convective flow in a rotating porous medium with mass transfer, Thermal science, 18, No.2(2014), 515-526. 
[13] M.Vidhya, R.Vijayalakshmi and A.Govindarajan, Chemical reaction effects on radiative MHD oscillatory flow in a porous channel with heat and mass transfer in an asymmetric channel, ARPN journal of engineering and applied sciences, 10, No.4(2015), 1839-1845.

[14] E.P.Siva, A.Govindarajan and V.Venkataraman, Unsteady Hydromagnetic Flow of Dusty Viscous Fluid in Rotating Channel with Varying Pressure Gradient, International Journal of Applied Engineering Research, 9, No.21(2014), 8875-8888.

[15] H.Haroun, Non-linear peristaltic flow of a fourth grade fluid in an inclined asymmetric channel, Computational Materials Science, 39, No.2(2007), 324-333.

[16] S.Srinivas, V.Pushparaj, Non-linear peristaltic transport in an inclined asymmetric channel, Communications in Nonlinear Science and Numerical Simulation, 13, No.9(2008), 1782-1795.

[17] M.Kothandapani, S.Srinivas, Non-linear peristaltic transport of a Newtonian fluid in an inclined asymmetric channel through a porous medium, Physics Letters A, 372, No.8(2008), 1265-1276.

[18] S.Nadeem, S.Akram, Influence of inclined magnetic field on peristaltic flow of a Williamson fluid model in an inclined symmetric or asymmetric channel, Mathematical and Computer Modelling, 52, No.1-2(2010), 107-119.

[19] M.Mishra, A.Ramachandra Rao and Z.Angew, Peristaltic transport of a Newtonian fluid in an asymmetric channel, Mathematical Physics, 54, No.3(2003), 532-550.

[20] T.Hayat, M.Iqbal, H.Yasmin, F.E.Alsaadi and H.Gao, Simultaneous effects of hall and convective conditions on peristaltic flow of couple-stress fluid in an inclined asymmetric channel, Pramana Journal of Physics, 85, No.1(2015), 125-148.

[21] E.P.Siva, A.Govindarajan, MHD Peristaltic motion under the influence of heat/mass transfer with chemical reaction through a porous medium in an asymmetric channel, European Journal of Scientific Research, 126, No.3(2014), 296-312.

[22] L.M.Srivastava, Peristaltic transport of a couple-stress fluid, Rheologica Acta, 25, No.6(1986), 638-641. 
[23] D.Pal, N.Rudraiah, R.Devanathan, A couple stress model of blood flow in the microcirculation, Bulletin of Mathematical Biology, 50, No.4(1988), 329-344.

[24] Kh.S.Mekheimer, Effect of the induced magnetic field on peristaltic flow of a couple stress fluid, Physics Letters A, 372, No.23(2008), 4271-4278.

[25] M.Vidhya, E.P.Siva and A.Govindarajan, MHD peristaltic flow of a couple stress fluids permeated with suspended particles through a porous medium under long wavelength approximation, ARPN Journal of Engineering and Applied Sciences, 10, No.7(2015), 3072-3077.

[26] S.K.Pandey, M.K.Chaube, Effect of magnetic field on peristaltic transport of couple stress fluids through a porous medium, Journal of Biological Systems, 19 No.2(2011), 329-344.

[27] S.Maiti, J.C.Misra, Peristaltic transport of a couple stress fluid:Some applications to hemodynamics, Journal of Mechanics in Medicine and Biology, 12 No.3(2012), 1-27.

[28] A.Alsaedi, N.Ali, D.Tripathi and T.Hayat, Peristaltic flow of couple stress fluid through uniform porous medium, Appl. Math. Mech. -Engl. Ed., 35 No.4(2014), 469-480.

[29] G.C.Sankad, G.Radhakrishnamacharya, Effect of magnetic field on the peristaltic transport of couple stress fluid in a channel with wall properties, International Journal of Biomathematics, 4, No.3(2011), 365-378.

[30] S.Hina, M.Mustafa, T.Hayat, A.Alsaedi, Peristaltic flow of couple-stress fluid with heat and mass transfer: An application in biomedicine, Journal of Mechanics in Medicine and Biology, 15, No.4(2015), 1-17.

[31] K.Ramesh, M.Devakar, Effects of heat and mass transfer on the peristaltic transport of MHD couple stress fluid through porous medium in a vertical asymmetric channel, Journal of Fluids, 2015 (2015), 1-19.

[32] M.Vidhya, K.Sundarammal, Laminar convection through porous medium between two vertical parallel plates with heat source, Proceedings of the International Conference on Frontiers in Automobile and Mechanical Engineering, 10.1109/FAME.2010.5714846, (2010), 197-200. 
